\title{
3.3 FILARIAL GENOMICS
}

\author{
Steven A. Williams
}

\section{Summary of Prioritized Research Needs}

1) Collecting materials

a) Before the opportunity is lost to preserve their genomes, collect geographically representative isolates of the various species and strains of the human filarial parasites,

2) Constructing libraries

a) Construct updated and additional genomic and cDNA libraries to represent completely the different stages and species of filarial parasites,

3) Sequencing

a) Expand expressed sequence tags (EST) sequencing from cDNA of a greater diversity of life cycle stages and parasites than currently available,

b) Complete the sequencing and annotation of B. malayi genome,

c) Expand the sequencing of $W$. bancrofti and $O$. volvulus genomes,

d) Complete the sequencing of the Wolbachia genome from $B$. malayi and mitochondrial genomes from $B$. malayi and $O$. volvulus,

4) Technical development

a) Develop transgenic methods useful for filarial parasites,

b) Use RNAi techniques for functional genomics studies,

c) Expand microarray assessments of gene expression with B. malayi and other filariae,

5) Repositories

a) Develop and maintain all collections of genomes, libraries, sequences, microarrays, etc. in central repositories accessible to all interested investigators within and outside the filariasis research community.

\subsubsection{Overview}

Genome projects were initiated for many species of parasites in the 1990s as part of a new approach to the study of these organisms that are among the most important human pathogens in tropical regions of the world. ${ }^{1}$ The WHO and the United Nations Development Program//World Bank/ WHO Special Program for Research and Training in Tropical Diseases (TDR) launched an initiative in 1994 to study the genomes of five of these parasites, including those that cause LF. The long-term goal of these genome initiatives has been to collect data that will both improve understanding of important biological problems such as parasite drug resistance, pathogenesis, and virulence and assist in the identification of new targets for chemotherapy and vaccines. ${ }^{1-3}$

\section{Why genomics for LF?}

For more than 10 years (approximately 1982-1994), standard molecular cloning techniques were applied to the study of filarial parasites (particularly B. malayi and $O$. volvulus), but few genes were cloned and identified. By the end of 1994, only 60 Brugia genes had been submitted to the Genbank database. It was clear that a new approach for studying the filarial genome was needed to make rapid progress in understanding the biology and biochemistry of these parasites. The genome project approach represented a complete departure from the way parasite genes had been studied in the past. Genome projects are typically not directed at the identification of individual genes, but instead at the identification, cloning, and sequencing of all the organism's genes.

At the first meeting of the Filarial Genome Project (1994), B. malayi was selected as the organism to be studied. This parasite was chosen over the other two medically important species of filarial parasites, $W$. bancrofti and $O$. volvulus, primarily because of the ready availability of all stages of the life cycle. ${ }^{4}$ In addition, molecular phylogenetic studies of filarial parasites had shown that all three of these species are closely related. Thus, much of the molecular data obtained on one species would be applicable to the other two. ${ }^{5}$

The genome of $B$. malayi $^{6}$ is about the same size (100 million base pairs) as that of the free-living nematode $\mathrm{Cae}$ norhabditis elegans. ${ }^{7}$ The number of protein-coding genes in C. elegans is estimated to be approximately 19,000 and the number of genes in Brugia is expected to be about the same.

\section{Filarial genomics: The 10 most important accomplishments to date.}

Filarial genomics now provide a basis for studying filarial parasites in ways that could not even be imagined only a few years ago. The 10 most important accomplishments of the first 10 years of filarial genomics include

1) the collection of sufficient numbers of parasites for the isolation of DNA and RNA from Brugia, Wuchereria, and Onchocerca from many locations around the world,

2) the construction of cDNA and genomic libraries for Brugia, Wuchereria, and Onchocerca,

3) sequencing many thousands of cDNA clones (EST sequencing): 24,000 from Brugia, 14,000 from Onchocerca, and 6,000 from Wuchereria,

4) clustering the EST data and developing user-friendly bioinformatics tools,

5) complete genomic sequencing of the B. malayi genome,

6) complete sequencing of the endosymbiont Wolbachia genome from Brugia and the mitochondrial genomes from Brugia and Onchocerca,

7) development of the first transgenic technique for getting DNA into a filarial parasite (B. malayi),

8) development of RNA interference (RNAi) technology for $B$. malayi and $O$. volvulus,

9) construction of the first microarray (gene chip) for a filarial parasite (Brugia malayi),

10) initiation of comparative genomics for a large number of nematode species (including C. elegans). 


\subsubsection{Research Needs}

Building on the 10 most important filarial genomics accomplishments.

\section{1) Collecting filarial parasites for the isolation of DNA and} RNA.

To construct the necessary cDNA and genomic libraries for the study of filarial genomes, parasites of various life cycle stages were collected from around the world. For B. malayi parasites were collected as $\mathrm{mf}$, second-stage larva (L2), L3, molting L3, L4, young adult, adult male and adult female stages. For $O$. volvulus, mf, L3, molting L3, adult male and adult female parasites were collected. For W. bancrofti, mf, L3, adult male and adult female parasites were collected. Laboratories contributing to this effort were from the United States, Egypt, Indonesia, Cameroon, India, French Polynesia, and others. This effort was time-consuming and costly, but absolutely essential; without the parasites, no work on the genomics could be undertaken.

For the future, a concerted effort must be made

- to collect large numbers of parasites of all stages of $W$. bancrofti and $O$. volvulus for future research needs; unlike B. malayi, there are no convenient animal models for these two species, so samples must be collected in the field; as elimination programs progress, it will become increasingly difficult to collect these parasites in sufficient quantity for research purposes,

- to collect geographic isolates of human filarial parasites from around the world and store them in a central repository; these parasites will be valuable for studying geographic variation and will prove especially important should drug resistance arise.

\section{2) Constructing cDNA and genomic libraries.}

Since fewer than 60 Brugia genes had been cloned by 1994, it was decided that the primary goal of the Filarial Genome Project would be the identification of at least 5,000 new Brugia genes. ${ }^{8}$ These new genes would help elucidate the biology of the organism and would aid in the identification of new vaccine candidates and drug targets. The plan was to identify these new genes by randomly selecting clones for DNA sequence analysis from new cDNA libraries that were to be constructed from all life cycle stages of $B$. malayi. The construction of many cDNA libraries from all of the life cycle stages would insure that a high proportion of the expressed genes of $B$. malayi would be represented. Such EST analysis is a rapid way to identify gene sequences, since no effort is made to completely sequence each cDNA clone.

Twelve Brugia cDNA libraries have been constructed in the bacteriophage lambda cloning vector ZAPII (Stratagene, La Jolla, CA) representing the following developmental stages: MF, L2, L3, molting L3 (L3M), L4, young adult (YA), adult male (AM), and adult female (AF). Unidirectional cloning was chosen to facilitate the use of these libraries in EST analyses. Seven of the Brugia cDNA libraries were constructed using conventional techniques, ${ }^{2}$ two were constructed using subtraction techniques, ${ }^{9}$ and three were constructed using PCR and the SL1 spliced leader sequence. ${ }^{10}$ The Brugia cDNA libraries (and cDNA libraries from $O$. volvulus and $W$. bancrofti) and all individual cDNA clones are available from the National Institute of Allergy and In- fectious Diseases/National Institutes of Health (NIAID/NIH) Filariasis Research Reagent Repository Center (FRRRC) at Smith College (contact genome@smith.edu or www. filariasiscenter.org). For the future,

- additional libraries need to be constructed to replace old libraries with better, more complete ones using methods and reagents that were unavailable when the original libraries were constructed,

- cDNA libraries also need to be constructed for life cycle stages that are not yet represented in the current collection.

\section{3) Sequencing cDNA clones (EST sequencing).}

By March 2004, there were already 26,215 ESTs containing more than 10 million basepairs of $B$. malayi sequence data submitted to the National Center for Biotechnology Information's dbEST database (www.ncbi.nlm.nih.gov/dbEST/ dbEST_summary.html). In addition, for O. volvulus, 14,974 ESTs have been submitted, while for W. bancrofti more than 6,000 ESTs are now available in the database. In total, this represents almost 20 million base pairs of expressed filarial parasite DNA sequences. All of these cDNA clones are available through the NIAID/NIH FRRRC at Smith College (contact genome@smith.edu or www.filariasiscenter.org). For the future,

- additional ESTs from W. bancrofti should be sequenced from a greater diversity of life cycle stages, because ESTs represent the most cost-effective approach to the identification of new $W$. bancrofti genes.

4) Clustering the EST data and developing user-friendly bioinformatics tools.

Since cDNA clones are selected randomly for sequencing and since duplication (especially of highly expressed genes) is unavoidable, the 26,000 Brugia EST represent approximately 8,945 Brugia clusters as determined by The Institute for Genome Research (TIGR) gene index (www.tigr.org/tdb/tgi/) and 8,392 as determined by the Nembase clustering ${ }^{11}$ (http:// nema.cap.ed.ac.uk/fgp.html). The number of genes represented by the EST data set is determined using a clustering algorithm that groups ESTs derived from the same gene into clusters. If the total number of genes in B. malayi is similar to that predicted for $C$. elegans, this data set includes almost $50 \%$ of all Brugia genes. A unified nomenclature for naming filarial genes and gene clusters is in place ${ }^{12}$ (http:// nema.cap.ed.ac.uk/fgn/funk/funkgenenames.html). Because these data are critical to gene identification,

- such bioinformatics-based analyses need to be expanded as additional ESTs are sequenced and as new clustering algorithms are developed.

5) Completing the genomic sequencing of the Brugia genome.

The complete genome sequence of $B$. malayi has recently been obtained through an NIH-funded project carried out at TIGR. ${ }^{13}$ The entire genome was sequenced using a whole genome shotgun strategy developed at TIGR. Approximately 1.3 million sequence reads have provided about $10 \times$ coverage (i.e., enough sequencing has been done to theoretically sequence the genome 10 times). This ensures that virtually all of the genome will be represented in the final sequence. Cur- 
rently, all of the genomic data is being assembled and gaps in the sequence are being closed. The genome is also being annotated by the filarial research community; a process initiated at the "Brugia malayi Annotation Jamboree" held for two weeks at TIGR during January 2004. For the future,

- additional effort is required to close the remaining gaps in the B. malayi genome sequence and to annotate the genome,

- sequencing of the W. bancrofti and $O$. volvulus complete genomes should be undertaken because of its benefit for the functional analysis of these genomes (including $B$. $m a$ layi) and its providing genetic clues of potential value for developing filariasis elimination strategies.

6) Completing the sequencing of the Wolbachia genome from $B$. malayi and of the mitochondrial genomes from $B$. malayi and $O$. volvulus.

Most filarial parasites contain an obligate intracellular bacterium most closely related to the Wolbachia intracellular bacteria found in mosquitoes and other arthropods. ${ }^{14}$ The genome was sequenced by a consortium of scientists led by New England Biolabs. ${ }^{15}$ Research over the past several years has shown that killing the Wolbachia bacteria results in reduced fecundity and often death of the filarial parasites. ${ }^{16-18}$ Since this intracellular bacterium contributes to the normal functioning of the filarial parasite, the bacterium itself is a target for novel chemotherapeutic and vaccination interventions. In addition, the complete sequence of the first mitochondrial genome from a filarial parasite $(O$. volvulus) was carried out at the University of Alabama Birmingham. ${ }^{19}$ The following year, the complete sequence of the B. malayi mitochondrial genome was completed at the University of Edinburgh. The mitochondrial genome provides additional targets for biological study and potential chemotherapy. What still remains are for

- the Wolbachia genomes from W. bancrofti and O. volvulus to be completely sequenced;

- the functional/comparative analysis of the Wolbachia genomes from all three species to provide potentially valuable information for LF and onchocerciasis elimination strategies.

\section{7) Developing a transgenic technique for Brugia.}

One of the principal barriers to rapid accumulation of knowledge on the biology and biochemistry of filarial parasites has been the inability of researchers to introduce DNA and RNA into the cells of these organisms. For example, the development of a transgenic technique (the ability to insert exogenous genes into the genome of the target organism) is important for the study of many organisms including bacteria, plants, and animals. Recently, a transgenic technique using a biolistic "gene gun" has been successfully applied to filarial parasites at the University of Alabama Birmingham. ${ }^{20}$ The development of this technique opens up the possibility of doing many gene function studies that were heretofore impossible in filarial parasites. Therefore,

- continued development of the "gene gun" and other transgenic methods for use with filarial parasites will be vital for using the genomic data now available to study gene function in these filarial parasites.

\section{8) Developing RNAi techniques for Brugia.}

Another important barrier to the rapid accumulation of knowledge on the biology and biochemistry of filarial parasites has been the inability of researchers to introduce RNA into the cells of these organisms. RNA interference has been used for the past several years in a wide variety of organisms as a means for silencing expression of specific genes. Such gene silencing is another valuable tool for studying gene function and for the identification of likely vaccine candidates and drug targets. This technique has been employed with great efficiency in $C$. elegans, a free-living nematode often used as a model system for filarial parasites. However, until recently, the technique had never been successfully applied to parasitic nematodes. However, in 2003 researchers at the University of Edinburgh successfully applied the RNAi technique in $\mathrm{B}$. $\mathrm{ma}$ layi adult female parasites, ${ }^{21}$ and in 2004 scientists at the New York Blood Center successfully applied RNAi to O. volvulus L3 larvae. Together, the development of a transgenic technique for introducing DNA and an RNAi technique for introducing RNA will dramatically revolutionize the way gene study can be undertaken in filarial parasites. Therefore,

- continued development of RNAi technology as a functional genomics tool, along with DNA transgenic techniques, will be vital for taking advantage of the new fully sequenced genome of B. malayi for functional genomics studies,

- RNAi should be used (as in C. elegans) as a screening tool for the identification of genes critical to the development of filarial parasites. Such genes encode products that may serve as excellent drug targets or vaccine candidates.

\section{9) Producing microarrays for filarial parasites.}

Another cutting-edge molecular biology technique now widely used in the study of genes in many organisms involves gene microarrays (gene chips). These arrays include thousands of genes on a single microscope slide or on a smaller silicon chip. These arrays enable the analysis of thousands of genes in a single experiment, instead of the more traditional analysis of one or a few genes at a time. Such technology has revolutionized the study of genes and gene expression. Brugia malayi microarrays have now been produced by a consortium of filarial researchers (led by scientists at Washington University and Smith College) at the Washington University Genome Center. These first microarrays for a filarial parasite are now available through the NIAID/NIH FRRRC at Smith College (genome@smith.edu or www.filariasiscenter.org). The arrays have been prepared using 65-mer oligonucleotides representing more than 3,000 genes of the parasite. It is still necessary for

- an updated version of the microarray to be prepared containing even more genes $(-5,000)$,

- sufficient numbers of parasites to be collected for performing the critical functional experiments necessary to understand filarial development, molting, response to drugs, etc.,

- the limited gene sequence data available for W. bancrofti and $O$. volvulus to be used in order to include these genes in subsequent versions of the filarial microarrays.

10) Initiating comparative genomics studies among nematodes (including $C$. elegans).

To take full advantage of the newly available, complete sequence of the genome of $B$. malay $i$ this sequence must be 
compared to those of other nematodes. Fortunately, the complete genomic sequence of the free-living nematode, $C$. elegans, is also available. In addition, more than 400,000 EST sequences are now available from more than 30 species of nematodes, largely through the efforts of scientists at the Washington University Genome Center and the University of Edinburgh. ${ }^{22}$ Such genomic and EST sequence data is essential for gene identification, for studies of the conservation of gene order (synteny), and for developing generalizations concerning gene function, gene regulation, and parasite-specific pathways. Because such comparative data will be critical for identifying new genes useful in developing vaccines and new drugs for filarial parasites,

- complete sequences need to be defined and comparative studies undertaken for the $W$. bancrofti and $O$. volvulus genomes (including their endosymbiont Wolbachia genomes).

\subsubsection{References}

1. Williams SA, Kemp DJ, 1996. Parasite genome projects. Meyers RA, ed. Encyclopedia of Molecular Biology and Molecular Medicine. New York: VCH Publishers, Inc., 306-312.

2. Williams SA, Johnston DA, 1999. Helminth genome analysis: the current status of the filarial and schistosome genome projects. Parasitology 118 (Suppl): S19-S38.

3. Degrave WM, Melville S, Ivens A, Aslett M, 2001. Parasite genome initiatives. Int J Parasitol 31: 531-535.

4. Unnasch TR, 1994. The filarial genome project. Parasitol Today 10: 415-416.

5. Xie H, Bain O, Williams SDA, 1994. Molecular phylogenetic studies on filarial parasites based on $5 \mathrm{~S}$ ribosomal spacer sequences. Parasite 1: 141-151.

6. Williams SA, Lizotte-Waniewski MR, Foster J, Guiliano D, Daub J, Scott AL, Slatko B, Blaxter ML, 2000. The filarial genome project: analysis of the nuclear, mitochondrial and endosymbiont genomes of Brugia malayi. Int J Parasitol 30: 411-419.

7. The C. elegans Genome Sequencing Consortium. 1998. Genome sequence of the nematode C. elegans. A platform for investigating biology. Science 282: 2012-2018.

8. Williams SA, 1999. Deep within the filarial genome: progress of the Filarial Genome Project. Parasitol Today 15: 219-224.
9. Diatchenko L, Lau YF, Campbell AP, Chenchik A, Moqadam F, Huang B, Lukyanov S, Lukyanov K, Gurskaya N, Sverdlov ED, Siebert PD, 1996. Suppression subtractive hybridization: a method for generating differentially regulated or tissuespecific cDNA probes and libraries. Proc Natl Acad Sci U S A 93: 6025-6030.

10. Scott AL, Yenbutr P, 1995. Molecular cloning of a serine protease inhibitor from Brugia malayi. Infect Immun 63: 1745-1753.

11. Blaxter ML, Aslett M, Guiliano D, Daub J, 1999. Parasitic helminth genomics. Parasitology 118 (Suppl): S39-S51.

12. Blaxter ML, Guiliano DB, Scott AL, Williams SA, 1997. A unified nomenclature for filarial parasites. Parasitol Today 13 . 416-417.

13. Ghedin E, Wang S, Foster JM, Slatko BE, 2004. First sequenced genome of a parasitic nematode. Trends Parasitol 20: 151-153.

14. Werren JH, 1997. Biology of Wolbachia. Annu Rev Entomol 42: 587-609.

15. Foster JM, Kumar S, Ganatra MB, Kamal IH, Ware J, Ingram J, Pope-Chappell J, Guiliano D, Whitton C, Daub J, Blaxter ML, Slatko BE, 2004. Construction of bacterial artificial chromosome libraries from the parasitic nematode Brugia malayi and physical mapping of the genome of its Wolbachia endosymbiont. Int J Parasitol 34: 733-746.

16. Townson S, Hutton D, Siemienska J, Hollick L, Scanlon T, Tagboto SK, Taylor MJ, 2000. Antibiotics and Wolbachia in filarial nematodes: antifilarial activity of rifampicin, oxytetracycline and chloramphenicol against Onchocerca gutturosa, Onchocerca lienalis and Brugia pahangi. Ann Trop Med Parasitol 94: 801-816.

17. Smith HL, Rajan TV, 2000. Tetracycline inhibits development of the infective-stage larvae of filarial nematodes in vitro. Exp Parasitol 95: 265-270.

18. Hoerauf A, Volkmann L, Hamelmann C, Adjei O, Autenrieth IB, Fleischer B, Buttner DW, 2000. Endosymbiotic bacteria in worms as targets for a novel chemotherapy in filariasis. Lancet 355: $1242-1243$.

19. Keddie EM, Higazi T, Unnasch TR, 1998. The mitochondrial genome of Onchocerca volvulus: sequence, structure and phylogenetic analysis. Mol Biochem Parasitol 95: 111-127.

20. Shu L, Katholi CR, Higazi T, Unnasch TR, 2003. Analysis of the Brugia malayi HSP70 promoter using a homologous transient transfection system. Mol Biochem Parasitol 128: 67-75.

21. Aboobaker AA, Blaxter ML, 2003. Use of RNA interference to investigate gene function in the human filarial nematode parasite Brugia malayi. Mol Biochem Parasitol 129: 41-51.

22. Parkinson J, Mitreva M, Hall N, Blaxter M, McCarter JP, 2003. 400,000 nematode ESTs on the Net. Trends Parasitol 19: 283-286. 